\section{Osseous Metaplasia of the Urinary Bladder: Mini Review of the Cases Reported After 1991}

\section{Abstract}

Osseous metaplasia of the urinary bladder is a rarely found condition by pathologists during histological analysis. Cancer, infection and inflammation are implicated with its appearance. Our paper examines cases after 1991, excluding those with osseous metaplasia in distal visceral and/or nodal metastases and osteogenic malignancies like osteosarcomas. A series of 11 cases were found after a thorough search in PubMed and Google Scholar databases, while "bladder osseous metaplasia" and "bladder bone formation" were used as key search terms. Urothelial - transitional cell carcinomas are found to be involved in $81 \%$ of the cases, while one case of angiosarcoma and one case of leiomyosarcoma are included with a mean age of 70.7 years. Only 2 of the subjects were females. The theories for its appearance speculate a subclone of tumour cells able to induce osteogenesis, transformation of undifferentiated stromal mesenchymal cells into osteoblasts and bone morphogenetic proteins BMP-2 and BMP-lb which act as cytokines. Osseous heterotopic metaplasia still constitutes a subject which needs further investigation.

Keywords: Bladder; Osseous metaplasia; Bone formation; Cancer

\section{Gregory Tsoucalas* and Aliki Fiska}

\author{
Department of Anatomy, Medical \\ School, Democritus University of Thrace, \\ Alexandroupolis, Greece
}

Corresponding author: Gregory Tsoucalas

” gregorytsoucalas@yahoo.gr

Department of Anatomy, Medical

School, Democritus University of Thrace, Alexandroupolis, Greece.

Tel: +302421078583

Citation: Tsoucalas G, Fiska A (2018)

Osseous Metaplasia of the Urinary Bladder: Mini Review of the Cases Reported After 1991. J Univer Surg. Vol.6 No.1:3

Received: January 09, 2018; Accepted: January 17, 2018; Published: January 19, 2018

\section{Introduction}

Osseous metaplasia in the urinary bladder may appear in several conditions, but mainly as an entity which accompanies bladder cancer. Tumours of the urinary bladder present the most serious cluster of malignant diseases of the urinary tract. They represent about $5-10 \%$ of all cancers globally. There is a preference towards male patients, with the ratio of male to female incidence being 3:1. Furthermore, there is a 39-fold higher prevalence of Caucasian males [1]. Environmental background plays a significant role. Thus, smoking, infections (schistosoma haematobium), race, gender, arsenic in drinking water, occupational exposure to aromatic amines (products of the chemical, dye and rubber industries as well as hair dyes, paints, fungicides, cigarette smoke, plastics, metals and motor vehicle exhaust), are all well known risk factors for bladder cancer [2,3]. Osseous metaplasia is a rarely observed lesion in the stroma of bladder carcinoma, either in the primary tumour and/or in a metastasis [4].

Our review aims to quote cases of osseous metaplasia of the bladder, alert pathologists to differentiate osseous metaplasia from osteosarcomas and gather the availliable knowledge on the subject.

\section{Methods}

A thorough search was conducted in the PubMed and Google Scholar databases, with the terms "bladder osseous metaplasia" and "bladder bone formation" as key words. Papers written in English and dated after 1991 were the only ones to enter our study. All manuscripts containing cases of cancer with osseous metaplasia inside the urinary bladder were included in our study. Cases with osseous metaplasia in distal visceral and/or nodal metastases and osteogenic malignancies like osteosarcomas were excluded.

\section{Results}

A series of 11 case reports matching our criteria were gathered (Table 1), available to be discussed [2,4-13]. Our search was limited concerning the time period, beginning in 1991, as Eble and Young in that year published a review of the literature. Only a few cases were reported after that [6]. Our findings support that osseous metaplasia of the bladder is found in cancerous environments. Urothelial - transitional cell carcinomas are mainly involved ( $81 \%$ of the cases). One case of angiosarcoma and one case of leiomyosarcoma are included. Excluding 2 paediatric 
Table 1 Cases of osseous metaplasia in the urinary bladder after 1991.

\begin{tabular}{|c|c|c|c|c|}
\hline S No. & Authors & Age & Gender & Histology \\
\hline 1. & Kral et al. [2] & 3 & Male & Bladder cancer - High grade urothelial carcinoma \\
\hline 2. & Rallabandi et al. [5] & 65 & Female & Bladder cancer - Angiosarcoma \\
\hline 3. & Boudabbous et al. [6] & 75 & - & Bladder cancer - High grade transitional cell carcinoma \\
\hline 4. & Cormio et al. [7] & 78 & Male & Bladder cancer - Urothelial bladder carcinoma, nodal metastases \\
\hline 5. & Gupta et al. [8] & 64 & Male & Bladder cancer - High-grade solid urothelial cancer \\
\hline 6. & Dudley et al. [9] & 66 & Male & Bladder cancer - High grade urothelial carcinoma \\
\hline 7. & Neşe et al. [10] & 60 & Male & Bladder cancer - Nested variant of transitional cell carcinoma \\
\hline 8. & Eble et al. [4] & 84 & Female & Bladder cancer - High grade urothelial carcinoma \\
\hline 9. & Mittal et al. [11] & 60 & Male & Bladder cancer - Low grade, noninvasive transitional cell carcinoma \\
\hline 10. & Skarda et al. [12] & 4 & Male & Bladder cancer - Urothelial high grade papillocarcinoma \\
\hline 1. & Majeed et al. [13] & 84 & Male & Bladder cancer - Leiomyosarcoma \\
\hline
\end{tabular}

cases, the mean age for the discovery of osseous metaplasia was 70.7 years of age. Only 2 of the subjects were females.

\section{Discussion}

Osseous metaplasia is a rare event in any tissue. While tumours' calcification is usually dystrophic and occurring in the areas of necrosis, osseous metaplasia, which refers to the formation of mature bone, has been demonstrated in benign and malignant conditions. Its incidence in malignant tumours is approximately $0.4 \%[14,15]$.

Osseous metaplasia is an additional, considered to bare benign prognosis, microscopic finding in bladder cancer. Bone formation in the stroma of a bladder tumour concerns the primary tumour and in the majority of the reported cases the tissue itself is too of benign appearance. Its main challenge is to differentiate it from sarcomatoid carcinoma with osteosarcomatous differentiation, or from radiation induced osteosarcoma, tumours that are a priori osteogenic and obviously change both the course and the treatment of the case [6].

Although the terms urothelial carcinoma and transitional cell carcinoma are synonyms, there is no global consensus and pathologists used them both. The configuration of the urothelial tumor's histological pattern is usually papillary, but it can also be of solid appearance or present mixed patterns. The lesion is most often seen as a thickening of the epithelium with an increased number of cell layers evenly distributed but densely packed. The cell nuclei often retain their normal orientation and round contour, while they are more pleomorphic and mitoses may be numerous. Papillary carcinoma is being presented as cauliflower or polypoid in shape tumour with superficial ulceration and necrosis and moderate architectural and cytologic atypia $[2,13]$. Angiosarcomas are an uncommon group of malignant neoplasms, characterized by rapidly proliferating, extensively infiltrating anaplastic cells derived from blood vessels, lining irregular blood-filled spaces [16]. Leiomyosarcomas are tumourus masses that originate from the smooth muscle cells, or precursor mesenchymal stem cells committed to this line of differentiation [17]. Despite the fact that both Angiosarcomas and Leiomyosracomas are classified as sarcomas, they are included in our study as they o not present a predisposition towards osseous metaplasia. All those malignancies may be diagnosed accompanied with osseous metaplasia of the stroma and should be differentiated from osteosarcomas, which are known to produce bone lesions, maybe through the expression of the bone morphogenetic protein receptor 2 [18].

Several theories are suggested for osseous metaplasia. The degree of calcification is reported to be minimal to extensive. Some speculate that a subclone of tumour cells able to induce osteogenesis could be created in a focus in the cancerous area, leading to completely benign osseous development, as transitional epithelium is a well-known bone-inducing agent $[6,19]$. Others propose the transformation of undifferentiated stromal mesenchymal cells into osteoblasts in a favourable environment, while it is widely believed that both bone morphogenetic proteins BMP-2 and BMP-Ib act as cytokines, target multipotent cells and induce differentiation of mesenchymal cells into osteoblasts, resulting to bone formation at heterotopic, extraosseous sites $[6,20]$. However, there are also several benign conditions that may microscopically present osseous metaplasia: inflammatory lesions and benign tumors, like ganglioneuroma, schwannoma, paraganglioma, hemangioma, and presacral teratoma [21,22].

The most typical sign of bladder cancer is a painless, gross hematuria, often as an isolated symptom. Computed tomography usually reveals calcification areas, while osseous metaplasia is confirmed after histological tumour biopsy examination, following curative transurethral tumour resection [2].

\section{Conclusion}

Osseous metaplasia in the bladder is a rare phenomenon. This is a feature which should be differentiated from the tumours able for bone production. Although this is a benign characteristic, metaplastic tissue procedures may be considered as precancerous lesions. Heterotopic osseous metaplasia in any tissue is itself a rare phenomenon, thus it should raise further examination and evaluation of its clinical significance for the patient. 


\section{References}

1 Torre LA, Bray F, Siegel RL, Ferlay J, Lortet-Tieulent J, et al. (2015) Global cancer statistics, 2012. CA Cancer J Clin 65: 87-108.

2 Kral M, Michalek J, Skarda J, Tichy T, Smakal O, et al. (2016) Highgrade urothelial bladder cancer in children: A case report and critical analysis of the literature. Biomed Pap Med Fac Univ Palacky Olomouc Czech Repub 160: 578-582.

3 Letasiova S, Medveova A, Sovcikova A, Dusinska M, Volkovova K, et al. (2012) Bladder cancer, a review of the environmental risk factors. Environ Health 11: S11.

4 Young RH, Eble JN (1991) Unusual forms of carcinoma of the urinary bladder. Hum Pathol 22: 948-965.

5 Rallabandi HB, Swain M, Gowrishankar S, Sinha S (2016) Postradiation angiosarcoma of bladder with extensive osseous metaplasia. Indian J Pathol Microbiol 59: 78-80.

6 Boudabbous S, Arditi D, Paulin E, Koessler T, Rougemont AL, et al. (2015) Ossifying metaplasia of urothelial metastases: original case with review of the literature. BMC Med Imaging 15: 30.

7 Cormio L, Sanguedolce F, Massenio P, Di Fino G, Selvaggio O, et al. (2014) Osseous metaplasia within a urothelial bladder cancer nodal metastasis: a case report. Anal Quant Cytopathol Histpathol 36: 117-119.

8 Gupta P, Hanamshetti S, Kulkarni JN (2012) Primary amyloidosis with high grade transitional cell carcinoma of bladder: a rare case report. J Cancer Res Ther 8: 297-299.

9 Dudley AG, Tomaszewski JJ, Hughes AH, Davies BJ (2012) Incidentally discovered osseous metaplasia within high-grade urothelial carcinoma of the bladder. Urology 79: e59-e60.

10 Neşe N, Kandiloğlu AR, Atesçi YZ (2007) Nested variant of transitional cell carcinoma with osseous metaplasia of the urinary bladder: a case report and review of published reports. Int J Urol 14: 365-367.

11 Mittal V, Rupala KK, Yadav R, Suryavanshi M (2017) Giant Sarcomatoid
Carcinoma with Osseous Metaplasia from Urinary Bladder Diverticulum. Ind J Surg Onc 8: 436-439.

12 Skarda J, Michalek J, Tichy T, Smakal O, Kral M, et al. (2015) High-Grade Non-Invasive Transitional Cell Carcinoma with Osseous Metaplasia of the 3-Year-Old Boy Urinary Bladder. J Clin Exp Pathol 5: 236.

13 Majeed SA, Ramesh K, Osman AR, El-Bashari YE, Al-Kowafi RM (1998) Leiomyosarcoma of urinary bladder with extensive osseous metaplasia. SMJ 19: 799-801.

14 Byard RW, Thomas MJ (1988) Osseous metaplasia within tumours. A review of 11 cases. Ann Pathol 8: 64-86.

15 Rallabandi HB, Swain M, Gowrishankar S, Sinha S (2016) Postradiation angiosarcoma of bladder with extensive osseous metaplasia. Indian J Pathol Microbiol 59: 78-80.

16 Young RJ, Brown NJ, Reed MW, Hughes D, Woll PJ (2010) Angiosarcoma. Lancet Oncol 11: 983-991.

17 Martin-Liberal J (2013) Leiomyosarcoma: Principles of management. Intractable Rare Dis Res 2: 127-129.

18 Wang S, Ren T, Jiao G, Huang Y, Bao X, et al. (2017) BMPR2 promotes invasion and metastasis via the RhoA-ROCK-LIMK2 pathway in human osteosarcoma cells. Oncotarget 8: 58625-58641.

19 Chalmers J, Gray DH, Rush J (1975) Observations on the induction of bone in soft tissues. J Bone Joint Surg Br 57: 36-45.

20 Komai Y, Morimoto S, Saito K, Urushibara M, Sakai K, et al. (2006) Possible involvement of bone morphogenetic protein 2 in heterotopic ossification in metastatic lesion from urothelial carcinoma of bladder. Int J Urol 13: 1126-1128.

21 Kawashima A, Alleman WG, Takahashi N, Kim B, King Jr BF, et al. (2011) Imaging evaluation of amyloidosis of the urinary tract and retroperitoneum. Radiographics 31: 1569-1582.

22 Varon C, Dubus P, Mazurier F, Asencio C, Chambonnier L, et al. (2012) Helicobacter pylori infection recruits bone marrow-derived cells that participate in gastric preneoplasia in mice. Gastroenterology 142: 281-291. 